\title{
Telephone consultations in primary care, how to improve their safety, effectiveness and quality
}

\author{
Muhammad Naseer Babar Khan
}

\begin{abstract}
Consultations on telephone are ever increasing especially in primary healthcare. Currently the estimated total number consultations in England rose from 224.5 million to 243.1 million in $1995 / 1996$ to 303.9 million to 313.6 million in $2008 / 2009$, out of which $3 \%$ of consultations in $1995 / 1996$ were telephone consultations which increased to $12 \%$ in 2008/2009. Literature search was done on published articles on telehealthcare which resulted in devising a telephone consultation model. An audit was carried out in urban Cambridge family practice over the period of one year after implementing this telephone consultation model. Following proposed consultation model by healthcare staff, it has improved patient satisfaction survey from $75 \%$ to $94 \%$, face to face consultation rate was reduced by $1.6 \%$, home visits were reduced by $2.9 \%$ however the direct economic saving could not be determined. Further research is required to assess the detailed economic analysis of using effective telephone consultation in healthcare. The data shown in this article is related to primary care in the UK, but its concept can be replicated by any country in Europe or rest of the world providing primary healthcare to public.
\end{abstract}

\section{Problem}

Primary care patients continue to use telephones to seek advice on a frequent basis. However, no system has been put in place to ensure that telephone consultations are being carried out as safely as possible to provide effective, safe and good quality healthcare to the patients. A recurring issue that is being brought to the attention of doctors is that patients call for a specific problem and reception staff attending the call were not able to identify whether they should be seen "immediately" or "urgently" or as a "routine" appointment. Despite best efforts, the patient satisfaction survey of the medical centre was never more than $75 \%$.

\section{Background}

Consultations on the telephone are increasing in primary care. The estimated total number of consultations in England rose from 224.5 million in 1995 to 313.6 million in 2009 , out of which $3 \%$ of consultations in 1995 were provided over the telephone which has then increased to $12 \%$ in 2009 (3). With increasing reliance on telephone consultations, healthcare delivery using this method is becoming more widely acceptable to members of the public and healthcare professionals. Since the invention and then increase of popularity of mobile phones, many prefer to seek professional advice while they are 'on the go'. One study has concluded that telephone consultations with GPs or nurses can safely substitute face-to-face consultations, although it is not clear that this reduced the number of face-to-face consultations over time. A third of people who had telephone consultation advice by a healthcare professional would have otherwise gone to a hospital emergency department $(1,2)$. However like any other communication skill, it requires training and up-skilling. Provided that healthcare staff have been trained in dealing with telephone consultations, it can improve patient satisfaction and reduce the rate of patient complaints. This can result in higher staff morale and reduce staff anxieties hence improving patient-healthcare relationship and experience.

\section{Baseline Measurement}

For this project, an audit was carried out on all the telephone consultations provided by healthcare staff over a 6 months period. The results were eye opening. 3780 consultations were carried out over the period of 6 months with an average of 630 consultations each month. It was found that there was very poor record keeping for telephone consultations. Safety netting which is vital for telephone consultations as it provides control to the patients so they are able to seek help if any further concerns develop, were only in place for $60 \%$ of consultations. There were also some patient complaints ranging from general dissatisfaction to potentially serious mistakes regarding the telephone consultation advice they received from healthcare team. Due to the absence of sensory input and visual cues of the caller, staff had increased anxieties in providing the advice and managing patient concerns on the telephone.

See supplementary file: ds2204.doc - "PDSA Cycle"

\section{Design}

After identifying the gravity of the problem, a literature search was carried out on tele-healthcare and a telephone consultation model was devised. Training was provided to all the staff involved in telephone consultations and followed by another audit to find out the results over the period of one year. The same audit proforma as before was used to assess the telephone consultations as to avoid bias. This audit proforma contained a structured patient and staff questionnaire. In the patient questionnaire, the aim was to find out if the identity of the caller had been established, if the patient had been satisfied with the advice given, if the patient was advised to call back if any further concern developed or was signposted to the 
appropriate service and also whether patient felt being able to better look after him or herself after the consultation. Record keeping of the telephone consultations was also looked at and whether staff felt more confident in dealing with consultation of this style.

\section{Strategy}

An appropriate telephone consultation model was already devised in light of the literature search. We have included it as a downloadable file. The staff providing the healthcare advice on telephone was appropriately given training on telephone consultations hence another audit was essential to find the outcome. The strategy involved carrying out the patient satisfaction survey regarding telephone consultation advice they received, checking of the record keeping, assessing patient complaints relevant to telephone consultation advice they received and also subjectively examining if staff felt more confident in dealing with telephone consultations after implementing this change. An audit confirmed improvement in staff confidence in dealing with telephone consultations, improved patient satisfaction and reduced face-toface consultations and home visits.

\section{Results}

The audit result confirmed that after providing training in telephone consultation to the healthcare staff and encouraging them to follow the telephone consultation model, it improved the patient satisfaction survey results from $75 \%$ to $94 \%$, face-to-face consultation rate was reduced by $1.6 \%$ and home visits were reduced by $2.9 \%$. The direct economic saving however, could not be determined. Further research is required to assess the detailed economic analysis of using effective telephone consultation in healthcare. From having only $60 \%$ of telephone consultations with safety netting in place, it went up to $99 \%$ of consultations. Finally record keeping of telephone consultations had been greatly improved.

See supplementary file: ds2203.doc - "Model Telephone Communication for Effective Consultation-1"

\section{Lessons and Limitations}

Lessons learnt during the completion of this project are as follows:

1. Clinical staff felt quite confident in dealing with telephone consultations after they were given a telephone consultation model to follow and they were given relevant training arranged by Royal College of General Practitioners. Such training is now easily available and can be arranged through local consortia.

2. There should be proper clinical governance structure to review telephone consultations by keeping voice recordings for training and quality purposes.

3. A regular audit is required to ensure that patients are receiving the best service and advice on telephone.

4. Patients like to use telephone to seek advice as it is quick, mostly cost effective and one can be 'on the go' while seeking expert medical advice.

There are some significant drawbacks and limitations of telephone consultations which one should always be wary of to avoid mistakes. For example there are no visual cues and it is very much dependent on the individual patients perception of the problem. If someone downplays the symptoms, it can lead to potentially life threatening mistakes. So it is vital to provide safety netting on the telephone. It is not a very good tool for those who do not speak the native language e.g. if patient does not speak English in English speaking country and healthcare worker attending the call does not speak the patient's language either then ideally professional interpreter should be arranged or such patients should be seen before providing any concrete medical advice.

\section{Conclusion}

A telephone consultation can provide safe, better quality and cost effective healthcare to patients but it is very easy to make mistakes on telephonic advice due to absence of visual cues hence the healthcare professionals should follow a proper structure of telephone consultation. Its cost effectiveness is still controversial in published papers, though some of the research papers have been included in this article showing cost effectiveness. More research is still required in this area. It is undoubtedly a convenient way of providing healthcare advice to patients. Listening attentively, using a well structured approach and knowing common pitfalls of this form of consultation would not only improve patient satisfaction but could also improve cost effectiveness indirectly. Training relevant to telephone consultations is available and should be sought by contacting local health authorities. Local guidelines on clinical governance of telephone consultations should be followed by the GP surgeries. Trainees should have supervised telephone consultations initially and trainer should at times listen to or read the transcript of trainees' independent telephone consultations to improve patient's safety and to identify trainee's learning needs if any. Telephone communication style can vary from individual to individual because we all have a different repertoire of skills which we utilise during an interpersonal interaction however following the structured consultation approach can provide better outcome. Although telephone consultation appears to have the potential to reduce GP workload, further rigorous evaluation of such systems is needed with emphasis on service use, safety, cost and patient satisfaction.

\section{References}

1. George S. (2002). NHS Direct audited: Customer satisfaction but at what price? British Medical Journal, 324:558-9

2. Hewitt H, Gafaranga J, McKinstry B. (2010).Comparison of face-to-face and telephone consultations in primary care: qualitative analysis. British Journal of General Practice, 60 (574):201-12

3. NHS information centre for Health and social care, (2009). Trends in Consultation Rates in General Practice 1995/1996 
to 2008/2009: Analysis of the QResearch ${ }^{\circledR}$ database.

\section{Declaration of interests}

None

\section{Acknowledgements}

Dr Z Alam (GP at Swineshead Medical Group, Swineshead, Lincolnshire, PE20 3JE),

Dr S Quadar (GP at Sleaford Medical Centre, Sleaford, Lincolnshire, NG34 7HD) 